\title{
A Case of Babesiosis Complicated by Hepatic and Renal Failure
}

\author{
Steven Krawitz MS IV, Nilay Kavathia MD, Pratik Choksy MBBS
}

\section{Case Report}

A 67-year-old male with a past medical history of chronic kidney insufficiency, splenectomy, and recurrent babesiosis infection was transferred to with jaundice and abdominal pain.

The patient initially presented to an outside hospital (OSH) with generalized weakness, chills, and gastrointestinal symptoms and was found to have Babesia on peripheral blood smears. The patient received seven days of azithromycin and atovaquone, followed by three days of clindamycin and quinine sulfate. The patient was discharged home with improved symptoms and a negative peripheral blood smear. Less than 24 hours later he returned to the $\mathrm{OSH}$ with worsening myalgias and abdominal pain. Physical exam was significant for jaundice and hepatomegaly. Laboratory data revealed anemia, elevated creatinine, bilirubin of $30 \mathrm{mg} / \mathrm{dL}$, and $1 \%$ parasitemia. At this point the patient was transferred for further management.

Patient's past medical history was significant for chronic renal insufficiency, congestive heart failure, type 2 diabetes mellitus, coronary artery disease, and hypertension. Previous surgical procedures included splenectomy after a motor vehicle accident, below the knee amputation (BKA) of the right leg, and cholecystectomy. Patient denied alcohol or substance abuse, and quit smoking tobacco approximately 10 years ago. He is a retired carpenter and denied any recent history of travel. Medications on admission included digoxin, clopidogrel, isosorbide mononitrate, carvedilol, sucralfate, esomeprazole, metoclopramide, amlodipine, octreotide, and insulin lispro.

On examination, patient looked fatigued, but in no acute distress. He was afebrile and had stable vital signs. Physical exam was significant for scleral icterus, ventral hernia and diffuse blanching, maculopapular rash on patient's hands and soles.

Patient was admitted and started on treatment with clindamycin and quinine. The patient's blood smear showed Babesia species with a $1.7 \%$ parasitemia (figure 1). During the hospital stay, patient had an acute mental status change with asterixis. Laboratory data obtained at that time revealed elevated INR, bilirubin, and ammonia levels. Given the clinical findings, patient was started on lactulose for possible hepatic encephalopathy. Hepatitis serology was found to be negative. Dialysis was initiated in the setting of acute renal failure. Patient's clinical status continued to worsen, so red blood cell exchange transfusion was initiated in an attempt to reduce the parasite load and help clear the infection. Despite the exchange transfusion and continued antibiotic treatment, the patient continued to have hemolysis, paracitemia, renal failure, and hepatic failure. At that point hospice discussion was initiated and patient was eventually transferred to hospice home care.

\section{Discussion}

Babesiosis is a tick born protozoan infection of red blood cells from the genus Babesia. The first human case was reported in $1957^{1}$. The four Babesia species that most commonly infect humans in decreasing order of incidence are B. microti, $B$. duncani, $B$. divergens, and $B$. venatorum. Babesiosis is a zoonotic infection requiring both an animal reservoir (usually mice) and a vector, which in humans is the Ixodid tick. The protozoan is transmitted through the saliva of a tick when it bites. Other possible modes of infection include perinatal transmission and blood transfusion.

The endemic areas for babesiosis show significant overlap with Lyme disease and ehrlichiosis ${ }^{2}$. The disease is most commonly seen in the northeast of the United States, and the most common cause of babesiosis in America is the B. microti species. While the majority of the early cases were seen in coastal areas near Massachsetts, babesiosis is now diagnosed with regularity in Rhode Island, Connecticut, and even New York and New Jersey ${ }^{3}$. Many cases have now been reported in other states stretching as far west as California and even Europe has confirmed cases of B. microti babesiosis.

The severity of babesiosis infection depends on the strain of parasite as well as host factors such as immune system status or asplenia. Clinically, patients infected with B.Microti typically fall into one of three categories of disease expression: asymptomatic, flu-like, and severe infection. Given the relatively high seroprevalence, many infected individuals are asymptomatic and unaware they carry the parasite $e^{4}$ One study estimates the prevalence of asymptomatic infection at about one third of infected individuals ${ }^{5}$. However, most infected individuals fall into the second category, mild to moderate flu-like illness. Similar to Lyme disease, up to two thirds of these symptomatic patients cannot recall getting bit by a tick, making diagnosis potentially difficult ${ }^{6}$. Patients typically present with malaise and fatigue and develop intermittent fevers with one of the following in order of prevalence: chills, sweats, headaches, anorexia, cough, nausea. Patients with severe disease more often present with malaise, arthralgia, myalgia, and shortness of breath often combined with thrombocytopenia and abnormal liver function ${ }^{7}$. Severe infection carries with it the potential of numerous serious complications including DIC, congestive heart failure, acute respiratory failure, liver and renal failure, and splenic rupture.

Diagnosis of babesiosis infection is typically made by microscopic examination and identification of the typical appearance of organisms on Geimsa stained thin red blood cell smears. Polymerase chain reaction (PCR) amplification, although more expensive, is more sensitive than microscopic identification and can be completed within one day. For diagnostic 


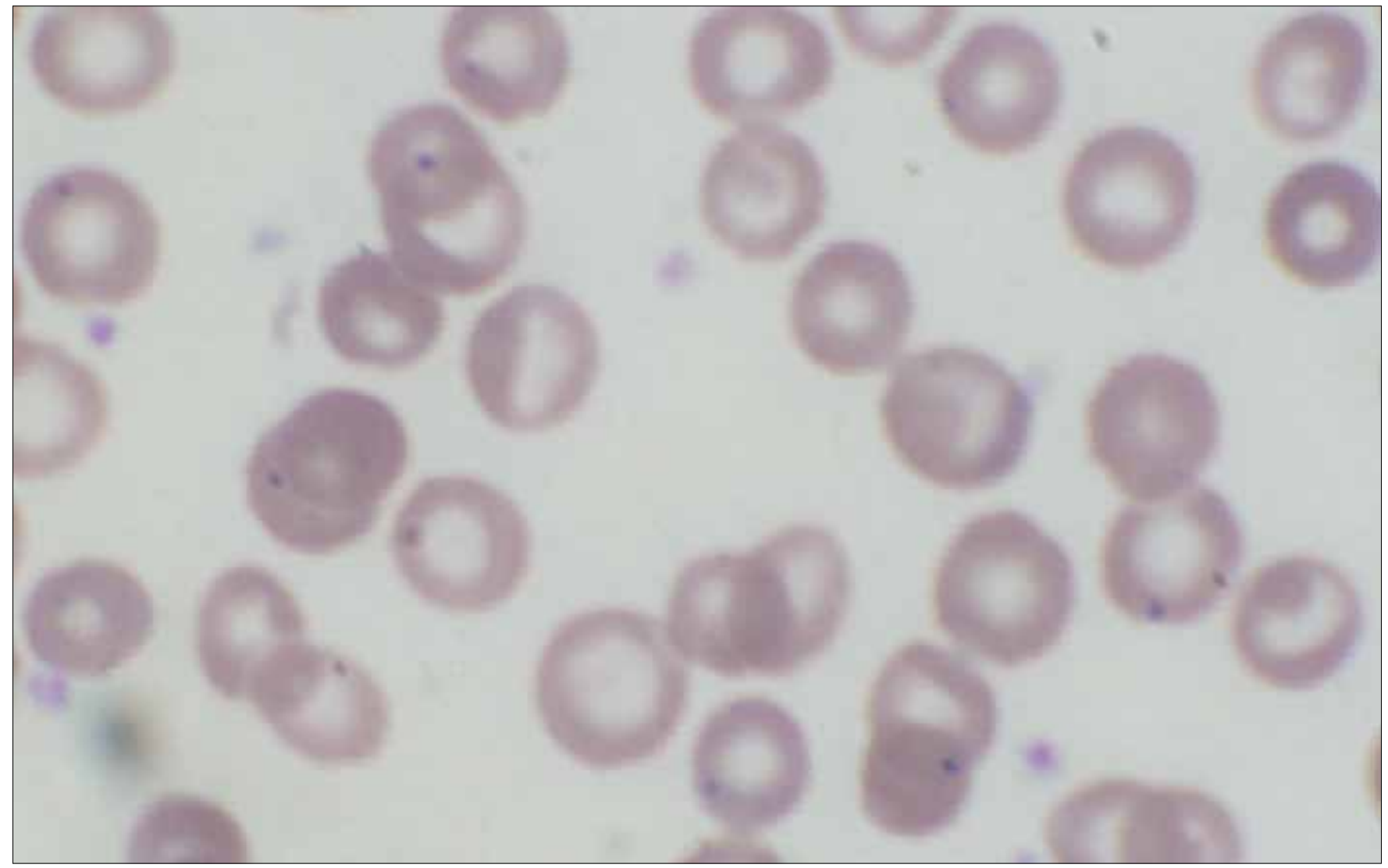

Figure 1. RBC peripheral smear showing typical babesiosis organisms in the patient's blood.

confirmation or when both microscopy and PCR are negative, serologic diagnosis can also be made. The standard treatments for babesiosis are the antibiotic regimens of clindmycin and quinine, or atovaquone and azithromycin, both administered for 7 to 10 days. Infections with a high parasitemia load or those that are persistent or relapsing might benefit from red blood cell transfusion to lower the parasite level in the blood. However, even with appropriate treatment, babesiosis can be fatal in certain patient populations.

Although most cases of babesiosis in the United States are mild to moderate or even asymptomatic, some cases are severe and may be fatal. It is important to be wary of the potential devastation the disease can have as well as who is more likely to manifest severe disease. The risk factors for severe disease include age older than 50, asplenia, coinfection with HIV or Borrelia burgdorferi, and an immunocompromised state. The case above demonstrates severe and resistant infection in the setting of multiple risk factors, namely increased age and asplenia. Though the patient was on appropriate antibiotic treatment and received multiple red blood cell transfusions, he still suffered from complications of the disease and was unable to clear the infection.

This case demonstrates that babesiosis is a potentially severe and fatal disease. It is important to recall the risk factors for disease severity, especially in certain at-risk populations. In treating such populations, potential end of life issues should be considered, as mortality and complications from the disease are significant.

\section{References}

1. Skrabalo Z, Deanovic Z. Piroplasmosis in man; report of a case. Documenta de Medicina Geographica et Tropica, 1957; 9(1):11-16.

2. Spielman A, Wilson ML, Levine JF, Piesman J. Ecology of Ixodes dammini-borne human babesiosis and Lyme disease. Annual Review of Entomology. 1985; 30:439- 460

3. Western KA, Benson GD, Gleason NN, Healy GR, and Schultz MG. Babesiosis in a Massachusetts resident. The New England Journal of Medicine. 1970; 283(16):854- 856.

4. Vannier E, Krause P. Update on Babesiosis. Interdisciplinary Perspectives on Infectious Diseases. 2009; Article ID 984568, 9 pages, 2009. doi:10.1155/2009/984568

5. Krause PJ, McKay K, Gadbaw J, et al. Increasing health burden of human babesiosis in endemic sites. American Journal of Tropical Medicine and Hygiene. 2003; 68(4):431-436.

6. Vannier E, Gewurz BE, Krause PJ. Human babesiosis. Infect Dis Clin North Am. $2008 ; 22: 469$.

7. Hatcher JC, Greenberg PD, Antique J, Jimenez-Lucho VE. Severe babesiosis in Long Island: review of 34 cases and their complications. Clin Infect Dis. 2001; 32:1117.

8. Krause PJ, Telford III SR, Spielman A, et al. Comparison of PCR with blood smear and inoculation of small animals for diagnosis of Babesia microti parasitemia. Journal of Clinical Microbiology. 1996; 34(11): 2791-2794. 